\title{
El empoderamiento de las mujeres versolaris en Euskal Herria
}

Gema Lasarte Leonet, María Teresa Vizcarra Morales, Andrea Perales-Fernández-de-Gamboa y

Ana Isabel Ugalde Gorostiza Euskal Herriko Unibertsitatea gema.lasarte@ehu.eus / mariate.bizkarra@ehu.eus / andrea.perales@ehu.eus / anaisabel.ugalde@ehu.eus

\section{RESUMEN}

A comienzos del siglo $\mathrm{XX}$, salvo raras excepciones, se conocían mujeres versolaris en el mundo del bertsolarismo. Datos de 2014 confirman que son mujeres el $48 \%$ de las participantes en escuelas infantiles de bertso, el $26 \%$ de las inscritas en las escuelas de adultos y el 20\% de las presentes en la plaza. En un siglo ha cambiado radicalmente la presencia de las mujeres en el bertsolarismo. Este artículo incide en los factores que han llevado a estas versolaris del silencio a cantar con una voz propia. Los motivos principales de estos cambios son la creación de la Asociación de Amigos del Bertsolarismo, el desarrollo de las escuelas de bertsos y el feminismo. Investigamos las iniciativas de estas versolaris y consideramos varias estrategias iniciadas por la Comisión de Género perteneciente a la Asociación de Amigos del Bertsolarismo. Las mujeres están movilizando un área masculina desde hace 200 años. Lo más notable es que en las últimas tres décadas, por iniciativa de las versolaris o por influencia de la Comisión de Género perteneciente a la Asociación de Amigos de Bertsolarismo, las versolaris están visibilizándose y empoderándose. Usamos el método cualitativo para esta investigación por medio de las entrevistas, y así se recogen 25 voces representativas de ese proceso de empoderamiento.

PALABRAS CLAVE

género; bertsolarismo; empoderamiento; mujeres 


\section{THE EMPOWERMENT OF BERTSOLARI WOMEN IN EUSKAL HERRIA}

\section{ABSTRACT}

At the beginning of the 2oth century in the world of bertsolarism, women performers of bertsos were unknown. Data from 2014 confirm that $48 \%$ of the participants in infant bertso schools, $26 \%$ of those enrolled in adult schools and $20 \%$ of those present at performances are women. Within a century, the presence of women in bertsolarism has radically increased. This article focuses on the factors that have enabled these bertsolaris to go from silence to singing with their own voice. The main reasons for these changes are the creation of the Association of Friends of Bertsolarism, the development of Bertso schools and feminism. We investigated the initiatives of these female bertsolaris and considered various strategies initiated by the Gender Commission of the Association of Friends of Bertsolarism. Women have played a role in this hitherto masculine domain for 200 years. The most remarkable thing is that in the last three decades, either due to the initiative of female bertsolaris or the influence of the Gender Commission, female bertsolaris are becoming more visible and empowered. We use the qualitative research method to conduct interviews with 25 representative voices in this process of empowerment.

\section{KEYWORDS}

Gender; bertsolarism; empowerment; women

\section{RESUM}

A començaments del segle XX, excepte rares excepcions, es coneixien dones bertsolaris al món del bertsolarisme. Dades de 2014 confirmen que són dones el $48 \%$ de les participants en escoles infantils de vers, el $26 \%$ de les inscrites a les escoles d'adults i el $20 \%$ de les presents a la plaça. En un segle ha canviat radicalment la presència de les dones en el bertsolarisme. Aquest article incideix en els factors que han portat a aquestes bertsolaris del silenci a cantar amb una veu pròpia. Els motius principals d'aquests canvis són la creació de l'Associació d'Amics del Bertsolarisme, el desenvolupament de les escoles de bertsos i el feminisme. Investiguem les iniciatives d'aquestes bertsolaris i considerem diverses estratègies iniciades per la Comissió de Gènere pertanyent a l'Associació d'Amics del Bertsolarisme. Les dones estan mobilitzant una àrea masculina des de fa 200 anys. El més notable és que en les últimes tres dècades, per iniciativa de les bertsolaris o per influència de la Comissió de gènere pertanyent a l'Associació d'Amics del Bertsolarisme, les bertsolaris estan fent-se visibles i s'empoderen. Fem servir el mètode qualitatiu per a aquesta investigació per mitjà de les entrevistes, $i$ així es recullen 25 veus representatives d'aquest procés d'empoderament.

\section{PARAUles CLAU}

gènere; bertsolarisme; empoderament; dones

REBUT: 2/O7/2O2I | ACCEPTAT: 25/O8/2O2I 


\section{El bertsolarismo y el género, el proceso de empoderamiento de las mujeres}

Las versolaris son sujeto de un proceso de cambio y paradigma de la transformación de todo un ámbito cultural. Han pasado de tener una voz subalterna, es decir, una posición sin identidad (Spivak I993), a tener agencia, como diría Kabeer (I993), a ser sujetos de su acción: del silencio a tener una voz propia. La agencia indica la capacidad para la acción, lo que en la narratología se denomina ser sujeto actante (Greimas I983). Las versolaris han ido empoderándose, pasando de ser objetos silenciados a sujetos activos (Rowlands I997). Hasta hace poco, el bertsolarismo no consideraba a las mujeres como sujetos activos, protagonistas o referentes canónicos. Por el contrario, las mujeres aparecían como objetos pasivos de amor y/o, en muchos casos, motivo de burla. Las «Ventajas de una mala dama», de Xenpelar, o «Tentar a una chica», de Txirrita, son un ejemplo de ello. Las metanarrativas tradicionales del bertsolarismo describían a las mujeres como desviadas del canon hasta principios del siglo Xxi. La perspectiva de género no surgió hasta que la sociedad, y con ella el campo del bertsolarismo (en ese orden, no al revés), se apoderó, entre otras cosas, de lo que ahora se reconoce como una verdad histórica: que a las mujeres se debe la primera práctica documentada, la del bertsolarismo medieval (Ariznabarreta 2020).

A pesar de que sus precursoras fueron silenciadas, bien es sabido que los grupos subalternos crean ideologías y acciones para mejorar sus condiciones. Y con las versolaris, a lo largo de los siglos, ha ocurrido lo mismo: sin dejar que sus voces se apagaran, unieron sus fuerzas en ocasiones para marcar sus propias estrategias (Larrañaga 200o). En ese camino se han ido marcando objetivos y estrategias (Lasa 2OIO).

Para reconocer esas estrategias se ha considerado importante entrevistar a veintiuna mujeres y cuatro hombres que viven el fenómeno del bertsolarismo. Entre estas voces, y a pesar de mantenerla en el anonimato, está Maialen Lujanbio, la primera mujer en ganar en 2009 ante I5.00o espectadores la txapela ${ }^{\mathrm{I}} \mathrm{del}$ campeonato de bertsolarismo.

Pero, primeramente, es necesario mencionar la relación entre bertsolarismo ${ }^{2}$ y género, así como la importancia que ha tenido el bertsolarismo para con la lengua vasca. El bertsolarismo ha estado unido, a lo largo de los siglos, al euskera y la cultura vasca. El bertsolarismo ha sido referente en la cultura vasca, tanto en la revitalización de la propia cultura como en el mantenimiento y las estrategias de supervivencia de la propia lengua. Se conoce el bertsolarismo desde el siglo XV, donde se documentan las primeras versolaris (Hernández 20I4; Larrañaga I995), pero estas fueron mujeres omitidas, invisibles. Por lo tanto, las versolaris de hoy día no vienen de un vacío, sino de una tradición que las ha condenado al silencio (Larrañaga I997; Agirre 20IO). Retomando a Wittig (I992), podemos afirmar que

\footnotetext{
I Los campeonatos se han realizado para poner al vencedor la txapela, y entre I936 y 2017 se han repartido I4. Solo una mujer la ha vestido: Maialen Lujanbio. En estos campeonatos se crean jurados con jueces, en su mayoría hombres, que evalúan todos los trabajos improvisados. El lenguaje, la métrica, la rima y los pies, la melodía, el texto, los recursos paratextuales; el tiempo, y la acogida del público son los ítems que puntúan.

2 Si bien mencionamos el bertsolarismo, nos referimos y analizamos más concretamente el bertsolarismo improvisado (Garzia, Sarasua y Egaña, 200I).
} 
las mujeres somos visibles como objetos sexuales, mientras que seguimos siendo invisibles como personas sociales, siendo el bertsolarismo ejemplo de esto. En consecuencia, el silencio secular de las versolaris ha hecho que las contemporáneas generen la necesidad de crear y afirmar sus identidades y discursos. Así, las versolaris de élite que han creado su propio discurso no han cambiado únicamente el sistema del bertsolarismo, sino el propio proceso creativo del verso. Alberdi (20I4) habla de la razón, la oratoria y la comunicación como características del bertsolarismo tradicional, mientras que las voces femeninas incorporan el mundo de las emociones, las vivencias interiores y la empatía. Esta fundamentación cambia el contexto del bertsolarismo. Como ejemplo de este cambió Hernández García (20I9) habla de sacudida emocional, de conmoción colectiva, que provocó Maialen Lujanbio en la final de 2017 con el verso que se anexa al final del artículo.

Mientras que el bertsolarismo estaba a punto de desaparecer, el bertsolarismo improvisado ${ }^{3}$ se ha convertido en un fenómeno de masas, lo que lo ha convertido en una herramienta indispensable para revitalizar, modernizar y difundir la lengua. Junto con esta masificación, también se ha dado la entrada masiva de mujeres, especialmente jóvenes, en el bertsolarismo (Agirre 20Io; Hernández 20I4). En el período de I970 a I990, mientras se creaban las escuelas de bertsos ${ }^{4}$, se cuestionaba si saldrían mujeres versolaris. Y las escuelas de bertsos, tres décadas después, han contribuido a reforzar la presencia de las mujeres y promover un bertsolarismo urbano y masivo.

En la literatura de mujeres, Showalter (I993) diferencia tres etapas: la primera, en la que las mujeres emularon las obras de los escritores; la segunda, con una protesta que clamaba a favor de la escritura de mujeres, y, por último, la actual, la que está en búsqueda y creación de la escritura propia. Las versolaris están llevando el mismo camino, pero para llegar a esa voz propia están negociando con la tradición y con el poder. Primero, a través de la emulación, y, después, generando disonancias. Estas disonancias han sido indicadores del malestar interno de estas mujeres, para después contemplar que estas discordancias externas son fuente del malestar externo (Agirre 20IO). En este momento, estas tres fases están presentes en el camino de las versolaris. Existen aquellas que emulan a los hombres, están aquellas que, a través de versos, formaciones o charlas desafían a esa hegemonía..., y están también aquellas que, tras un largo camino, han logrado una voz propia.

Las mujeres versolaris se han juntado e iniciado su proceso de empoderamiento en las últimas tres décadas. Por un lado, han generado encuentros e iniciativas (Begiristain 2OII). Por otro lado, la presencia, discurso y praxis de las versolaris de élite las han llevado a convertirse en referentes de muchas versolaris (Lasarte et alii 20I6). Finalmente, han estado negociando en espacios masculinamente hegemónicos, tanto en la sociedad como en la Asociación de Amigos del Bertso-

\footnotetext{
3 El bertsolarismo improvisado consiste en expresiones culturales nunca antes reproducidas frente al público. Se han conocido improvisadoras alrededor del mundo (cubanas, mexicanas, colombianas...) que trabajan acompañadas de un instrumento musical. Las versolaris ejecutan su acto improvisado sin música, pero es cantada con una melodía concreta y a capela. La melodía establece la métrica, es decir, el número de sílabas; sin embargo, la versolari no cuenta las sílabas, sino que las acomoda dentro de la melodía (Garzia, 200I; 20I2). 4 En las escuelas de bertsos se enseña a cantar versos a la gente. Para ello hay profesorado y material.
} 
larismo $^{5}$ (de aquí en adelante, la Asociación). Además, se ha creado la Comisión de Género y en todos los ámbitos de acción se está incorporando la perspectiva de género (Erkiaga 2008). Así, las acciones emprendidas por las versolaris, los discursos de las versolaris de élite y, por último, la Comisión de Género le han dado altavoz a la voz subalterna silenciada por el discurso hegemónico. Defendemos el concepto de empoderamiento de Murgialday (2013) en tanto que entendemos que se empoderan los agentes faltos de poder y para ello lideran su propio empoderamiento. Las mujeres versolaris, en ese sentido, han marcado las directrices estructurales de cómo trabajar la perspectiva de género en todo el sistema del bertsolarismo. Han insertado el género en la cultura del verso (véase el anexo I), la canción sin género recogida por Gómez Urda (202I).

\section{2. ¿Qué es el bertsolarismo improvisado o canto improvisado?}

La base del bertsolarismo es la improvisación. Para Lekuona (I974), el bertsolarismo es una subsección de la literatura oral, una literatura que no se escribe. También ha sido denominada literatura popular o poesía popular. En la descripción de Lekuona, el bertsolarismo tiene las siguientes características: la rapidez mental, el carácter dialectal y la fuerza de la espontaneidad (Larrañaga Arriola 20I3). La influencia de Lekuona hizo que hasta finales del siglo xx el bertsolarismo se contemplase como una subsección de la literatura de Euskal Herria, siendo así recogido por la mayoría de investigadores. El propio Mitxelena (I988), al hablar de la literatura popular, comenta que esta es mayormente oral (Larrañaga Arriola 20I3). Lekuona ubicó el bertsolarismo dentro del género oral, subrayando la característica de la improvisación. Así, describió cómo en apenas unos segundos el versolari utiliza la técnica de atrás hacia delante: piensa en lo último que va a decir y organiza su verso desde ahí, la característica más propia de la improvisación (Larrañaga Arriola 20I3). Las versolaris de élite, empero, han cambiado notablemente la creación de versos. Ahora, en vez de crear sus versos sobre un final consistente, apuestan por dejar finales abiertos. Esto nos recuerda a la mayor renovación hecha en la narrativa literaria: el cambio del final. Así optan por finales sugerentes, abiertos, que en muchas ocasiones dan que pensar al público. Es un estilo que caracteriza a las versolaris de élite, que impregnan su cantar con temáticas y estructuras que invitan al público a reflexionar constantemente sobre su cosmovisión.

A pesar de que para comienzos del siglo xx el bertsolarismo era mayormente escrito (existían las hojas de versos), para finales de siglo se fue afianzando la improvisación, el bertsolarismo con público, similar al que se conoce hoy en día.

Existe otra modalidad. Son los bertsos escritos [...]. La técnica es la misma, pero el resultado más elaborado, pues el autor o autora no repentiza ante el público [...]. Hace un tiempo se los conocía como hojas de bertsos, bertsos nuevos o inéditos y hojas de canciones. Solían venderse y gozaban de gran aceptación. La gente se los aprendía de memoria y se transmitían de generación en generación. Escritos o improvisados, los bertsos siempre pueden ser cantados, porque han sido pensados con ese fin (Ugalde 202O: I42).

5 La Asociación de Amigos del Bertsolarismo se creó el I8 de junio de i987. Se generó en tres ámbitos: la organización de la transmisión, la promoción, y la difusión de saberes. Hoy día están en marcha los departamentos de transmisión, promoción e investigación y comunicación. Tiene 2.500 socios y cerca de 70 personas trabajan en la organización. 
La improvisación no es un producto creado de la nada, sino que es fruto de la renovación de una tradición que se está adaptando a los nuevos tiempos. Aierdi (2007) considera que el bertsolarismo es un acto moderno con raíces en la tradición, ya que la gente lo toma como cultura popular. Aierdi menciona que estas definiciones muestran algunas de las características del bertsolarismo: la tradición, la cultura popular o la modernidad, entre otras. Lo que queda de manifiesto es que el bertsolarismo es una tradición con raíces en la cultura popular, con lo que hay que ver su relación en ese molde cultural (Larrañaga Arriola 2013).

Teniendo en cuenta este cambio, Larrañaga Arriola (2013) dice que el bertsolarismo actual ha pasado de ser una actividad masculina a una en la que también caben las mujeres. Esto hace que esa hegemonía masculina se diluya al contar con una mayor presencia femenina en las plazas. Hasta hace dos décadas el número de mujeres era bastante reducido. Según Larrañaga (I995), han sido los valores masculinos los que más se han estimado en el bertsolarismo: razonamiento, entendimiento, palabra, pensamiento. Mientras tanto, a las mujeres se las ha construido desde el sentimiento, el cuidado y el amor, siempre de acuerdo con la construcción social tradicional (Larrañaga Arriola 20I3). Además, la diferencia entre lo escrito y la improvisación ha aumentado la brecha, lo que nos hace afirmar lo que comenta González Abrisketa a propósito de las mujeres pelotaris: «[...] el espacio central de la cultura vasca, aquel que corresponde al bertsolari, al político, al plaza-gizon, el espacio de la plaza» (20I3: 86). Por lo tanto, la presencia de la mujer en la escena pública resulta un cambio de paradigma en el escenario del bertsolarismo.

\section{La historia del bertsolarismo}

Lekuona decía que el bertsolarismo había surgido con el pastoreo, «que tenía aspecto neolítico y prehistórico» (Barandiaran 2OII: I5). Esta antigua tradición se remonta, como mínimo, al siglo XV, ya que Mitxelena (I988) ubica en esa época las baladas más antiguas: «También son de época las mujeres que Lekuona creía que improvisaban y las eresiac. También ubicaba ahí a las profazadoras, las mujeres unidas a la palabra» (Hernández 2006: 62).

La antigüedad del bertsolarismo, sin embargo, no generó demasiado interés entre los investigadores, ya que hasta el siglo xx había una opinión negativa hacia esta entre los literatos e historiadores: «Juego insignificante e inútil entre vagabundos y no estudiosos, costumbre desinteresada y contraria a la moral, baile suelto o amoroso como la trikitixa» (Barandiaran 20II: I5). En los años treinta del siglo xx hay un punto de inflexión, ya que, tal y como el propio Lekuona afirma, el bertsolarismo ya no era solo una cuestión de bares, sino que era la voz del pueblo, y enfatizó, entre otras, la perfección de sus formas, el rápido movimiento entre imágenes, las elipsis y sus conmovedoras expresiones.

Le debemos a Lekuona la estimación actual por los versos; igualmente, I960 también es importante, ya que en esta época la versificación comienza a callejear, socializar e incluso se teoriza sobre el tema. Y, entre otros versolaris, un cura removió las aguas. Fue Amuriza quien, con sus versos y sotana, renovó el bertsolarismo, precisamente porque teorizó en torno al tema. Igualmente, no se puede olvidar a Joxerra Garzia, ya que propuso un marco teórico propio para la improvisación. Para Garzia (200I; 2OI2), el bertsolarismo requiere de una formulación teórica 
nueva, ya que «el objetivo de la improvisación no es crear textos de gran valor literario sino generar emociones en el público» (Garzia 20I2: 40). Quiere alejar la improvisación de la poética, «ya que, desde el prisma de la poética escrita, la mayoría de versos improvisados son de escasa calidad» (Garzia et alii 200I). Además, la improvisación no se puede someter a la escritura, ya que el verso no se agota en el texto, es también canto (melodía, voz) y se une con el público porque «un verso improvisado no es nada si no está contextualizado» (Garzia et alii 200I: I40). Así, sitúa la improvisación más cercana a la retórica que a la literatura y propone una definición más precisa del bertsolarismo improvisado: «[...] el bertsolarismo es un género retórico oral epídico, cantado e improvisado» (Garzia et alii 200I: I79).

La necesidad de aunar la teorización y el verso han contribuido en virtud del bertsolarismo y, para ello, las escuelas de bertsos han sido espacios necesarios (Artetxe 2020). Sarasua comenta que la creación de las escuelas de bertsos enriqueció la cantera de versolaris, al activar a organizadores, aficionados, facilitadores de temas $^{6}$, alumnado y profesorado (Barandiaran 2OII). La primera escuela de bertsos se creó en la Ikastola Almen y ahí se formó la primera versolari: Loidi’. Mardaras afirma que, si las escuelas de bertsos no existieran, tampoco habría mujeres versolaris (Mardaras, en Barandiaran, 2OII). Mardaras ${ }^{8}$ fue la primera mujer de la etapa contemporánea que participó en un campeonato de bertsos en 1985.

Si la sotana de Amuriza fechó la innovación en la historia del bertsolarismo, la falda de Kristina Mardaras puso otra marca, la del género. Como mencionamos anteriormente, en la historia hegemónica del bertsolarismo no existen mujeres; siempre han estado, pero han sido invisibles hasta hace poco (Hernández 20I4). Se han movido en ámbitos privados (la casa, las amistades) (Larrañaga I997). Con todo, Lekuona recordó que la supervivencia y transmisión del bertsolarismo, así como el disfrute, se les debe a las mujeres (Larrañaga 1995). He ahí el paradigma de la invisibilidad y el olvido, por un lado, y ser la raíz más profunda de la versificación, un oxímoron como tal:

Así pues, esta particular «homeostasis» se resuelve en que el bertsolarismo deviene un paradigma de la masculinidad, y se equilibra en el olvido de la contribución de las mujeres al bertsolarismo. Para rescatarlas de este olvido hay, pues, que recurrir a recuerdos familiares e íntimos... es que en la intimidad de lo doméstico se recuerda y se sitúa a las mujeres en el quehacer bertsolarístico (Larrañaga I995: 4IO).

En ese quehacer se proponen los siguientes objetivos:

I. Identificar las necesidades de las versolaris en los procesos de empoderamiento.

2. Analizar las dinámicas y los problemas encontrados por la Comisión de Género a la hora de introducir la perspectiva de género. Hay que considerar los pasos dados para visibilizar las voces subalternas.

6 Se encargan de moderar los recitales de versos y proponen los temas y las métricas que van a abordar los y las versolaris.

7 Arantzazu Loidi ha ganado varios campeonatos en las escuelas de bertsos, pero, a diferencia de otras contemporáneas, su fina voz le generó muchas críticas.

8 Ha habido diversos campeonatos en el bertsolarismo, en las escuelas, escritos, pero el principal es en el que los y las versolaris más importantes compiten. La única mujer que ha ganado ha sido Maialen Lujanbio, en 2009 y 2017. 
3. Dilucidar los pasos que tendrán que dar las versolaris para lograr una igualdad efectiva.

\section{Método}

La metodología que se utiliza es el análisis de datos o contenidos cualitativos, procesados por el programa NVivo I2. Se toman como herramienta metodológica hegemónica los grupos de discusión, teniendo en cuenta que el corpus entrevistado agradece poder hablar libremente, y que la técnica del debate facilita la recogida de testimonios. Además, se adjuntan notas de campo de las investigadoras que recogen la información que escapa a la transcripción de los grupos de discusión y revelan información en torno al ambiente que se genera en el encuentro. Por otra parte, las personas que no pudieron participar en los grupos de discusión elaboraron relatos escritos, respondiendo, así, el mismo cuestionario que siguieron los grupos de discusión. En los grupos de discusión los concurrentes se retroalimentan al escucharse entre ellos. Se les dio total libertad a las voces para responder como quisieran. Así, quisimos destilar también los pensamientos y sentimientos surgidos de la realidad de las versolaris y aficionadas. De esta forma, se abogó por crear una relación horizontal entre las participantes y las investigadoras. Es decir, se optó por disminuir el poder de la investigadora en aras de beneficiar la investigación y generar un acto comunicativo real, tal y como afirma la teoría (Barbour 20I3; Bodgan y Biklen I982; Colás y Buendía I992; Flick 2OI4; Goetz y Lecompte I988). Siguiendo a Limón y Crespo (2002), en todas las discusiones la moderadora incidió en que la discusión será siempre moderada y respetuosa.

\subsection{Participantes}

Esta investigación se ha desarrollado en el marco del bertsolarismo y se ha consultado a agentes pertenecientes a esta tradición (versolaris, investigadores, expertos, aficionados, jóvenes de las escuelas de bertsos, facilitadores de temas y participantes de la Comisión de Género).

De este modo, se crearon cinco grupos de discusión: el primero se hizo con tres mujeres versolaris (experimentadas y expertas); el segundo y tercer grupo se hicieron con jóvenes aficionados, donde participaron cinco mujeres en el segundo grupo y tres hombres en el tercero (todos miembros de las escuelas de bertsos, más concretamente, los responsables de los campamentos de verano); el cuarto grupo incluía tres mujeres versolaris y una facilitadora de temas, y en el quinto grupo participaron dos mujeres y un hombre de la Comisión de Género. Por otro lado, algunas versolaris y otras expertas en el área que no pudieron participar en los grupos de discusión nos hicieron llegar su relato: recibimos cinco. En total son 25 personas, 2I mujeres y 4 hombres. Asimismo, participan 9 investigadoras (8 mujeres y i hombre). La información se ha recogido de diversas fuentes. Así, las notas de campo se han denominado LOr y LO2; los grupos de discusión, ETI, ET2, ET3, ET4 y ET5, y los relatos, ErI, Er2, Er3, Er4 y Er5. Además, marcamos a las mujeres con $\mathrm{E}$ al final y a los hombres, con $\mathrm{M}$. 


\section{2 Herramienta para la recogida de datos}

Las herramientas usadas para la recogida de información han sido los grupos de discusión y sus transcripciones, las notas de campo tomadas por las investigadoras y los relatos. Los grupos de discusión son conversaciones en grupo, con elementos de las entrevistas en profundidad. Así, se contrastan y detectan las opiniones de diversas personas a la par que se recogen opiniones, sentimientos y contradicciones. Aquí, el tema ha sido la situación del bertsolarismo y el género a través de un planteamiento comunicativo y dialógico (Habermas I994; Aubert, García y Racionero 2009).

Rapley (20I4), por su parte, señala que en un grupo de discusión que tiene por objeto la recopilación de datos no existe una respuesta definitiva, a no ser que estas se provoquen razonablemente en función del contexto y de las manifestaciones con otros interlocutores. En relación con la toma de notas de campo, como han puesto de manifiesto varios autores, para que la observación y la comprensión de la realidad sean más globales, el hecho de que participe más de un investigador permite intercambiar diferentes puntos de vista sobre el momento en que vivieron (Rekalde, Vizcarra y Macazaga 2OII; 2OI4).

\subsection{Procedimiento}

Estos grupos de discusión se han complementado con un investigador dinamizador, y dos investigadores que han trabajado en la toma de notas de campo, además de un cuarto investigador que se ha encargado de lo audiovisual para poder posteriormente realizar transcripciones. El análisis de la información, junto con el trabajo de campo, constituye una etapa importante (Goetz y Lecompte I988). En nuestro caso, el análisis de la información recibida ha sido un proceso, fundamentalmente inductivo-deductivo, dinámico y sistemático, que ha requerido conocer, seleccionar, clasificar, comparar e interpretar el mensaje de las opiniones recibidas. Este proceso ha permitido extraer las ideas principales (dimensiones, categorías) de las informaciones representadas como conceptos que configuran los fenómenos (subcategorías) (Coffey y Atkinson 2003; Rodríguez, Gil y García I996). Como ya se ha indicado, los contenidos se clasifican en categorías y han sido revisados por varios investigadores del grupo antes de empezar a redactar el informe. Así, usamos el sistema categorial que presentamos más adelante. Hemos creado diferentes dimensiones para clasificar los datos obtenidos y se han interpretado los resultados tras el tratamiento de estos a través de NVivo I2. Una vez analizados todos los resultados se redacta el informe y se extraen las principales aportaciones y/o conclusiones.

\subsection{Herramienta: sistema categorial}

Este sistema categorial puede considerarse como una herramienta de análisis. Como se ha extraído de los testimonios de los participantes. 


\begin{tabular}{|c|c|c|}
\hline Dimensiones & Categorías & Subcategorías \\
\hline \multirow{4}{*}{$\begin{array}{l}\text { Los procesos de } \\
\text { empoderamiento de las } \\
\text { mujeres: las acciones } \\
\text { iniciadas, además de la } \\
\text { asociación. }\end{array}$} & \multirow[t]{4}{*}{$\begin{array}{l}\text { Las necesidades de las } \\
\text { mujeres: necesidades } \\
\text { expresadas por las mujeres. }\end{array}$} & $\begin{array}{l}\text { Toma de conciencia: condición } \\
\text { indispensable para realizar el } \\
\text { proceso de empoderamiento. }\end{array}$ \\
\hline & & $\begin{array}{l}\text { La falta de implicación de los } \\
\text { hombres ha sido notoria hasta } \\
\text { ahora. }\end{array}$ \\
\hline & & $\begin{array}{l}\text { Acciones e iniciativas. } \\
\text { Características de los encuentros. }\end{array}$ \\
\hline & & $\begin{array}{l}\text { Necesidad de alianzas: conciencia } \\
\text { de crear red. }\end{array}$ \\
\hline & \multirow{5}{*}{$\begin{array}{l}\text { El trabajo de la Asociación de } \\
\text { Amigos del Bertsolarismo, y } \\
\text { el trabajo de la Comisión de } \\
\text { Género. }\end{array}$} & $\begin{array}{l}\text { Función principal: el papel general } \\
\text { de la Asociación. }\end{array}$ \\
\hline & & $\begin{array}{l}\text { Miedos y actitudes negativas: } \\
\text { resistencias hacia la mirada de } \\
\text { género. }\end{array}$ \\
\hline & & $\begin{array}{l}\text { Formación. A quién se dirige. } \\
\text { Jueces, facilitadores. }\end{array}$ \\
\hline & & $\begin{array}{l}\text { Formación. Tres lineas: se ha } \\
\text { trabajado en tres áreas (mujeres, } \\
\text { hombres, mixto). }\end{array}$ \\
\hline & & $\begin{array}{l}\text { Formación. Escuela de Bertsos } \\
\text { Feminista: o escuela de } \\
\text { empoderamiento para las } \\
\text { mujeres. }\end{array}$ \\
\hline
\end{tabular}

Tabla I. Herramienta de análisis.

Este proceso ha permitido extraer las ideas principales (dimensiones) de las informaciones representadas como conceptos determinantes de los fenómenos (categorías) (McMillan y Schumacher 2005; Rapley 20I4). Además, este procedimiento no se ha utilizado como sistema de interpretación, sino que ha adoptado una perspectiva hermenéutica en la que se quería comprender el sentido de la comunicación entre los seres humanos. Las voces transcritas se han clasificado en categorías. El sistema categorial cumple una doble función: por un lado, sirve para el análisis de textos, y, por otro, para organizar la discusión del apartado de resultados (véase la tabla I).

\section{Resultados}

\subsection{Las necesidades de las mujeres}

En este apartado se muestran los resultados de los datos procesados que dan cuenta de las necesidades que han percibido las personas entrevistadas en el mundo del verso para empoderar a las mujeres. Parten de comentar que se ha tenido en cuenta lo que se ha hecho y lo que se está haciendo para introducir la perspectiva de género y el feminismo en el mundo del verso. 


\subsubsection{La toma de conciencia}

El bertsolarismo tradicional no ha sido un ejemplo muy atractivo para muchas mujeres versolaris, por lo que las mujeres han cuestionado el paradigma tradicional del bertsolarismo y han reconocido que otros modelos son posibles. «Muchas de las iniciativas actuales son a título personal, otras se han decidido como colectivo, pero la cuestión fue visibilizar y validar todas» (I5O223_ET5_M).

En este sentido existe un cambio en cuanto a los modelos femeninos en el escenario, sobre todo porque al principio muchas imitaban a los hombres en el mundo del verso. Afortunadamente, se han ido reivindicando otro tipo de ejemplos. «Mi experiencia comenzó en Navarra, había mujeres referentes, pero en ese momento ellas entraban dentro del modelo de versolari masculino» (I5O223_ LOET5_M).

Así, las reivindicaciones de las mujeres han sido muy importantes, tanto para agitar las conciencias como para sensibilizar sobre el tema. Así lo comentan algunas versolaris:

[...] a ver, si aquí se está haciendo un trabajo, será porque habrá en qué trabajar (I5OI3I_ET3_E).

[...] somos la mitad de la sociedad, pero mira lo que hay en el campeonato, en los campeonatos escolares, tendríamos que hacer una apuesta estratégica para los próximos años (I5OIO9_ETI_E).

Una cosa es cómo lo valoremos nosotras, creo que muchas lo agradecemos, valoramos esta perspectiva de género dentro de este ámbito cultural (I5OIO9_ETI_E).

\subsubsection{La falta de implicación de los hombres}

En el bertsolarismo, como en la sociedad, se nota la falta de implicación de los hombres en la inserción de la perspectiva de género, ya que algunos ni se acercan.

[...] los hombres no se mojan, no solo que no se han mojado, ni siquiera se acercan a reconocer esto, o a decir «Jo, pues podemos ir por ahí, o deberíamos de profundizar en esto», a ver... (I5OIO9_ETI_M).

Es muy difícil crear un grupo para perder tus privilegios individuales, eso no se hace. Se mueven las que están subordinadas, siempre se van a movilizar las minorías. Los hombres serán aliados, pero pocos serán activistas (I5O223_LOET5_M).

Ya que los versolaris no conocen ninguna iniciativa de género concreta, $y$, como ocurre, en otras áreas, los espacios de valoración social tienen escasa presencia de mujeres.

Estoy a favor de iniciativas con perspectiva de género en el bertsolarismo. Pero no sé si eso se da hoy día. En los espacios laborales de mayor honor y relevancia escasean las mujeres (I50306_Er2_E). 


\subsubsection{Acciones e iniciativas}

Emergen diversas iniciativas al hablar de las acciones emprendidas por las mujeres versolaris, entre ellas, los primeros bertso-encuentros de mujeres en Azkizu en 2003, y que se repitieron en 2013, o el Día del Bertso de 20I4, donde se eligió el género como tema central. Sobre Azkizu, una versolari comenta cómo tuvieron suerte con el momento, ya que la intención era juntar a mujeres improvisadoras de todo el mundo para analizar cómo se podía insertar la perspectiva de género.

En Azkizu nos reunimos personas de 30 países para ver cómo insertar la perspectiva de género (I5O223_LOET5_E).

Y yo he tenido contacto con la Comisión de Género desde que empezamos con la movida de Azkizu y, de alguna forma, es nuestro contacto con la Asociación (I5OIO9_ETI_E).

Azkizu sirvió para reflexionar, a través de la experiencia, sobre muchas cosas. Al comienzo pensaban que la discriminación les ocurría a otras, pero después vieron que era cosa de todas.

Yo, por ejemplo, antes de ir a Azkizu, nunca hubiera imaginado la necesidad de difundirlo al resto (I5OI3I_ET2_E).

[...] pues, por ejemplo, me ha servido para tomar conciencia y reflexionar, [...] fijarme en lo pequeño y ver cómo influye, y yo ahí veo la influencia de la Comisión de Género hacia nosotras (I5OI3I_ET3_E).

La unión unánime de las mujeres con el género fue el Día del Bertso de 2014. Cuando se organizó el evento, el tema estaba relacionado con el género, algo polémico, aunque las valoraciones fueron muy positivas.

Por eso quiero decir que el Día del Bertso de 2014 fue muy potente, supuso una sacudida tremenda, ¿no? Se ve que hay un trabajo tremendo ahí y joer... (I5OI3I_ET2_M).

Otro momento en el que se trabaja el género es en los campamentos. En los campamentos $^{9}$ se emplean dinámicas y metodologías innovadoras, pero es difícil cambiar las dinámicas machistas, ya que están muy enraizadas en la sociedad.

A pesar de hacer dinámicas y trabajar estos temas diariamente, se ven conductas machistas inconscientes (I5OI3I_LO_I).

En este proyecto hay mujeres de todas las edades y se valora socialmente. Sus experiencias en el mundo del bertsolarismo se han convertido en un espacio para compartir, y se sienten cómodas con el respaldo del grupo, creando conciencia y redes.

El habernos juntado y compartido ha abierto un espacio en el bertsolarismo que antes solo se conseguía mediante las escuelas de bertso. Poder juntarnos cuando estamos aisladas y sentirte protegida... (I5O223_ ET5_E).

Se hace un gran trabajo de género en el bertsolarismo. Este trabajo genera charlas, encuentros y talleres, algo que ayuda a las jóvenes versolaris.

9 Las profesoras de bertso se mueven por Euskal Herria como monitoras en los campamentos. Miles de niños se acercan en verano y el objetivo es pasarlo bien mientras que aprenden a improvisar. 


\subsubsection{Las alianzas}

Se considera indispensable la alianza entre versolaris.

[...] las alianzas son indispensables. Pero no solo las alianzas entre mujeres, sino también entre hombres y mujeres, y sentirte valorada emocional e intelectualmente (I5OIO9_ETI_E).

El grupo habla de redes, alianzas, si no existiese esa red no saldrían a la plaza. Es un proceso (I5OIO9_LOIETI_E).

Las versolaris sienten la protección de colegas profesionales. Pero ese camino no ha sido fácil, porque antes han tenido que descubrirse personalmente y desde la teoría feminista. Todavía queda camino para conseguir una conciencia feminista.

Creo que todavía tenemos mucho trabajo en este tema. Contradicciones, miedos, envidias, juicios [...], tenemos que trabajarlos para lograr una verdadera conciencia feminista (I50306_Er3_E).

Entre las versolaris existe protección, pero valoran aún más la que reciben fuera de este mundo; en el movimiento feminista, amistades, familia... El mundo del verso es competitivo y dicen que los juicios dentro de este son los más duros.

En el mundo del verso, como en los otros, hay una gran pelea de egos, es un ejercicio público y eso tiene sus consecuencias... (I5O3O6_Er3_E).

Es indispensable trabajar la autoestima y equilibrar el interior. Porque es un sistema inmisericorde (I50306_Er3_E).

En los ámbitos públicos, como el bertsolarismo, son necesarias alianzas de diversa índole, para crear la conciencia feminista de grupo y para sentirse protegida. Además, la competitividad hace que las envidias, juicios y miedos se manifiesten. Estos elementos no ayudan en la construcción de la conciencia feminista. Hasta ahora se ha trabajado mucho y se ha traído el tema al centro, pero queda mucho por hacer. Para seguir adelante, son necesarias las escuelas de bertso feministas y las iniciativas que salgan de estas.

Está claro que, en el bertsolarismo, como en otros espacios, la mayoría es masculina. Para poder seguir adelante hay que tener cuidado y mucha conciencia para no dar un paso atrás. De vez en cuando, como en el Día del Bertso, estas iniciativas funcionan para conocer la impresión del público.

\subsection{La Asociación y la Comisión de Género}

\subsubsection{Funciones de la Asociación}

Considerando el trabajo de la Asociación, se resalta su función principal: influir en las sesiones, que los diferentes agentes hagan su trabajo. La Asociación pretende fomentar un ambiente sano, promoviendo materiales didácticos e investigaciones, y se encarga de la formación, por lo que no se podía plantear una formación sin tener en cuenta el género. «La asociación no se mete a organizar o a dar plazas, es un camino a largo plazo el que se requiere para sanear el ambiente» (I5O223_LOET5_E).

Cuando se comenzó a sanear ese ambiente, se puso el foco en las relaciones, $\mathrm{y}$ vio el género como una clave que tener en cuenta: «Para mejorar las relaciones 
el género es clave, y para ello hay que insertar la perspectiva de género» (I5O223_ LOET5_M).

Así, la Asociación quiso tratar los problemas en torno al género creando una Comisión de Género. Al comentar los quehaceres de la Comisión de Género, se subrayaron primeramente los objetivos a cumplir, siendo uno de ellos la asesoría desde la perspectiva de género. «Nosotras estamos ahí para promover la perspectiva de género, para que cada uno después lo lleve a su terreno» (I5O223_ET5_E).

La Comisión de Género es la única que tiene asesoría en género, a diferencia del resto de departamentos. Tienen claro que no son un grupo feminista y que su prioridad es cuidar el bertsolarismo. Una de las funciones principales de la Comisión de Género es ayudar a la mujer en el escenario, para que se sienta empoderada en él.

Queremos tener influencia ayudando a la mujer en el escenario, para que cada vez más se sumen. Saber qué claves hay que tocar para lograr el empoderamiento, las teorías del cuerpo y el trabajo psicológico son claves (I5O223_LOET5_E).

Otra función es visibilizar a las mujeres, poner en valor lo que ellas hacen, y que en ese reto cada departamento tenga su responsabilidad. La Comisión de Género quiere dejar claras cuáles son las responsabilidades departamentales.

El reto de los próximos diez años es que cada departamento coja su responsabilidad real (I5O223_ET5_E).

Para mí dentro de la Asociación se le ha dado fuerza a la Comisión de Género o, por lo menos, un reconocimiento mínimo al ver que nos juntamos en masa. No sé, ¿eh? Vieron de repente que «estas dos son capaces de juntar a cuarenta personas para pasar un fin de semana», y pensaron, «aquí algo hay», y es ahí donde yo veo la fuerza, en el grupo (I50Io9_ ET_E).

Se ve la necesidad de la Comisión de Género porque se ha hecho un trabajo interno y se busca la reflexión. Es útil para cambiar algunas ideas interiorizadas, para marginar comportamientos machistas y para tomar decisiones.

¿Que qué ha traído la creación y trabajo de la Comisión de Género de la Asociación? Creo que, entre todas, hemos traído el tema al centro. Hace diez-quince años no estaba formado el discurso, no tenía fuerza, y era más fácil que cada uno culpara de sus dificultades, enfado, a sus frustraciones o límites personales, porque solo veíamos violencia estructural (I50306_Er3_E).

Yo, viendo esa Comisión de Género, sé que nos ha aportado mucho y me ha ayudado a hacer un trabajo interno importante para limpiar la cabeza y guardar las ideas que hay que guardar y creo que eso es lo más importante, y de ahí mirar a fuera y ver qué se puede hacer (I5OI3I_ET2_E).

\subsubsection{Miedos y actitudes negativas}

El mayor miedo de la Comisión de Género es crear comportamientos contrarios, ya que si algo se pone de moda en el mundo del bertsolarismo se desprestigia inmediatamente. Por ello tienen que tener cuidado. 
Cuando algo está de moda no nos gusta..., entonces hay posibilidades de que se cuestione, y vienen los chistes cada vez que se mencione la palabra género y se dejará el trabajo hecho, por lo que prefiero que seamos más sutiles. Tenemos que estar ahí, pero no como tema (I5O223_ET5_M).

Siempre han temido no conseguir el efecto buscado y lograr lo contrario, y así, en vez de integrar la perspectiva de género, convertir esto en un motivo de broma. Ven la necesidad de difundir la perspectiva de género a todos los ámbitos.

¿Qué es trabajar el género en esta creación cultural? ¿Qué hay que hacer? Igual piensas que un tema es a favor del género y el efecto es el contrario... La incorporación de la perspectiva de género no es trabajo exclusivo de la Comisión de Género, estamos dentro de la Asociación, pero también hay muchas personas con influencia (I5O223_ET5_M).

Se han organizado talleres de formación con facilitadores de tema, jueces, periodistas ${ }^{\mathrm{IO}}$, donde se ha incidido en la necesidad de no caer en estereotipos sexistas a la hora de proponer temas, al igual que tener ciertos factores en cuenta a la hora de puntuar.

[...] y entonces lo hicimos, no fue un gran plan..., pero dijimos, bueno, pues para formar a los facilitadores, a los jueces y los periodistas, vamos a dar unas sesiones, y, entonces, el tema está presente, y así, cuando vas a organizar cosas, pues ese tema ya se ha trabajado (I5O223_ET5_E).

\subsubsection{Comisión de género: formación}

Entre las versolaris surgen dudas en cuanto a la formación en género, ya que no tienen claro si los facilitadores y los jueces la reciben. Se sobreentiende que tienen sensibilidad de género, pero igual la practican fuera del bertsolarismo.

En las reuniones de facilitadores y jueces, pues, recibirán alguna formación en género, pero creo que, además, deberían de formarse también fuera...(I5OI3I_ET3_E).

La Comisión también se ha reunido con directivos de cadenas de televisión, buscando la reflexión interna.

Pues, por ejemplo, visibilizar las biografías, un departamento de investigación, dar unas becas para investigar, están los campeonatos, se han dado ideas (I5O223_ET5_E).

Pero hay que promover el cambio en el escenario:

[...] hasta que no veamos a la abuela cantando en el escenario esto no se va a normalizar, pero eso en el bertsolarismo, en la coeducación, en la literatura, arte, pintura, en toda área que sea parecida (I5O223_ET5_E).

[...] no somos políticas, no queremos cambiar el plan en cuatro años, queremos que esto funcione $y$, dentro de 750 años..., quizá tenemos otro bertsolarismo, otras plazas, otros recitales... (I5O223_ET5_M).

Io En el universo del bertso, además del versolari, también son muy importantes los facilitadores de temas, jueces y periodistas, y, si eso fuera poco, debería incluirse también a los agentes culturales y al público. Es impensable formar a todos. 
El género se está teniendo en cuenta en diversos ámbitos, se extiende el mensaje. Además de en las escuelas y campamentos, los facilitadores y los jueces también están reflexionando.

Y ¿los facilitadores y los jueces? ¿Las escuelas y campamentos? No controlo tanto esos ámbitos, pero por lo que me cuentan se está trabajando en todas las áreas, más o menos. El chip del «género» se está difundiendo en todas las áreas y se están realizando iniciativas concretas. Se están haciendo esfuerzos (I5O306_ErI_E).

Cuando pensaron en la Comisión de Género hacer una formación, se comenzó sin ningún plan, sin ninguna guía.

Experimentar, no hay un camino concreto, pero la Comisión de Género está experimentando, entonces experimentamos en diferentes líneas y, con el tiempo, estas se mueren o se activan (I5O223_ET5_E).

\subsubsection{Las tres líneas en la formación}

Han creado tres líneas de formación: la primera, para empoderar a la mujer, la segunda, para sensibilizar al hombre, y la tercera, mixta, para que hombres y mujeres trabajen juntos. Cada línea lleva una velocidad diferente, un nivel de activación distinto. A las mujeres no hay que convencerlas, ellas saben que tienen que seguir trabajando. Las otras líneas son más lentas, pero crearán su propia metodología.

Solo las mujeres forman parte de esa formación y el empoderamiento es su objetivo (I5O223_ET5_M).

Hay que trabajar con los promotores y por eso hemos creado las tres líneas, una con mujeres, para que conozcan qué trabajo se está haciendo, para reflexionar sobre el trabajo a hacer con los jóvenes. También pensamos en nivelar a la gente a través de su conciencia de género, pero, como hay ritmos de aprendizaje diferente, se han inventado tres caminos: uno para conseguir el empoderamiento femenino, otro para conseguir la sensibilización masculina, y el mixto, para crear relaciones y vernos entre nosotras (I5O223_LOET5_M).

La línea para las mujeres está mejor organizada. Necesitan formación, pero ya hay gente formada en el grupo y que es muy activa. Se han generado encuentros y talleres, y se abren nuevos caminos constantemente.

No tenemos nada que hacer en la formación de mujeres, no tienes que decir nada, ellas se organizan. La línea de mujeres da resultados, tenemos encuentros anuales, los cursos que da Virginia Imaz. Ahora estamos buscando cosas nuevas (I5O223_ET5_E).

La segunda línea es la de los hombres. Aquí se quiere sensibilizar. Al comienzo quería copiarse lo que se hacía en la línea de las mujeres, hasta que vieron que eran dos colectivos distintos, con necesidades y características diferentes. Queda claro que algunos hombres no se sentían cómodos en ese modelo tradicional de bertsolarismo. 
Hicimos el intento de imitar la línea de los hombres con las mujeres y es otra cosa, tenemos que seguir investigando, a pesar de que sea difícil mover a los clásicos (I5O223_ET5_E).

A veces se habla de las nuevas masculinidades, otras, de las sensibilidades, y en otras, del despertar de la conciencia.

La conciencia, entender la situación, género, familias, el bienestar interno, los hombres como tema (I5O223_ET5_E).

El tercer camino es la línea mixta, saben que para que se dé un cambio en la sociedad se necesita la colaboración de todos.

[...] hay que reforzar el mixto, hombres y mujeres están mezclados, y hay que ver cómo hacer y a dónde ir. Es necesario ver el trabajo hecho hasta ahora para seguir adelante [...]. Como asociación, nuestro trabajo es coger el tema del género y difundirlo, queremos trabajar el género en la línea mixta para recibir las opiniones de ambas partes, para saber qué hacer. Se quiere hacer una especie de diagnóstico en el mixto, y ver qué camino hay, el camino del próximo año, dar el salto a la plaza (I5O223_LOET5_E).

\subsubsection{La bertso eskola feminista}

Se quiere crear una escuela de bertsos feminista, sin demasiado ruido, para que las mujeres se sientan cómodas en el escenario y en el campeonato, para que tengan salidas hacia ciertos temas.

Se quiere crear la Escuela de Bertsos para mujeres, pero sin publicidad, para no generar presión en las mujeres. Es un trabajo importante, cada departamento tiene que responsabilizarse desde la perspectiva de género y para ver qué se puede hacer (I5O223_LOET5_M).

Se le llama la Escuela de Bertsos Feminista. A pesar de haber sido creada con ganas, convencimiento y fuerza, no la quieren publicitar. Es una idea mediáticamente innovadora, pero no quieren sacar rédito por el miedo a las consecuencias.

[...] es un caramelo mediático, pero no queremos decir qué metodología se usa, no queremos sacar a las mujeres al escenario con esa presión (I5O223_ET5_E).

[...] noté a mucha gente desconfiada a mi alrededor diciendo: «Vais en contra de vuestro discurso», porque nosotras queremos que en la plaza seamos iguales, pero luego vamos solas por nuestro lado (I5OI3I_ET3-E).

[...] se quieren dar los inicios hacia una escuela de bertsos feminista y crear material y compartir un poco qué modelo queremos (I5OI3I_ET3_M).

Tanto la creación de las escuelas de bertsos como la Comisión de Género también han servido para reflexionar sobre todo el proceso de empoderamiento de las mujeres versolaris sobre las necesidades, sobre la propia conciencia, como las resistencias.

Uxue Alberdi, Maialen Lujanbio, Ainhoa Agirreazaldegi..., han dado charlas y apoyado las escuelas de bertso feministas..., y ahora se ha puesto en marcha el espectáculo El secreto del Bertsolarismo [...], el ambiente está caldeado (I5O306_Er5_E). 
1. Trabajo de la Asociación de Amigos del Bersolarismo: Fomentar el bersolarismo
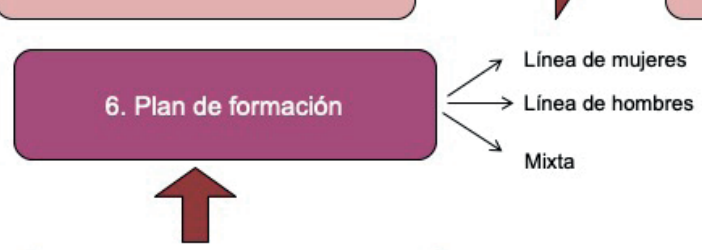

Acciones: Encuentro de 2013, género como tema el Día del Bertso 2014, talleres de formación, pautas para material didáctico, Escuela de Bertsos de mujeres
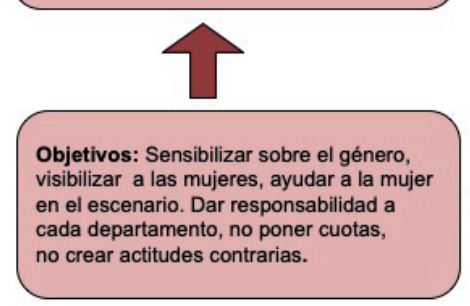

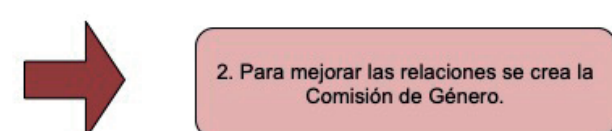
Comisión de Género.

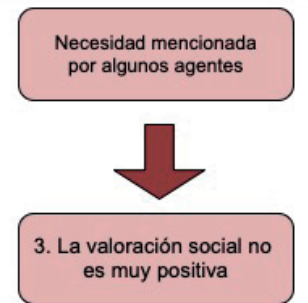

4. Falta de implicación masculina

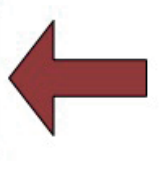

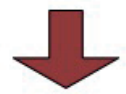

5. Tareas de la Comisión de Género

Figura I. Actividades emprendidas por la Asociación de Amigos del Bertsolarismo.

\section{Conclusiones}

Respecto al primer objetivo, inferimos que las versolaris han hecho un gran trabajo en su proceso de empoderamiento, pero estas necesidades y estrategias parten de las mujeres (Rowlands i997). Así, la Asociación, viendo la preocupación de las mujeres, decidió poner en marcha la Comisión de Género en 2009.

En relación con el segundo objetivo, la Comisión de Género ha hecho trabajo de asesoramiento desde el comienzo, pero tiene como objetivo cuidar el bertsolarismo y ayudar a las mujeres en la salida al escenario, ya que muchas no se atreven a salir a la plaza o al campeonato. Quiere visibilizar y valorar a las mujeres, pero tiene miedo de generar el efecto contrario. La investigación muestra cómo, al comienzo, muchas versolaris imitaban a los hombres cantando (Showalter I983), pero, con el trabajo, ese modelo ha cambiado en los últimos veinte años. Es decir, la mujer en el bertsolarismo ha pasado de ser objeto a sujeto (Greimas I983), a tener un discurso propio, y es por ello que en nuestra investigación las versolaris jóvenes agradecen el trabajo hecho, a pesar de que la implicación venga mayormente de las mujeres. Un dato que confirma esta realidad es que en la actualidad la campeona de versolaris es mujer: Maialen Lujanbio. También, las campeonas de Vizcaya y Navarra son mujeres, Onintza Enbeita y Saioa Alkaiza respectivamente. Y aunque en Guipúzcoa Beñat Gaztelumendi es el txapeldun, la final resultó muy disputada entre Alaia Martín y Gaztelumendi. Este dato es muy importante porque las mujeres versolaris comprenden el $20 \%$ del colectivo. Solo en Álava y en la parte vascofrancesa, los campeones son hombres. Otro dato es el estilo de Maialen Lujanbio, un estilo todavía por investigar que empieza a tener 
seguidores. Las propias versolaris señalan la necesidad de analizar en profundidad la obra de Maialen Lujanbio para precisar los aspectos realmente innovadores que aporta a la improvisación. Este cometido es de interés en relación con el tercer objetivo en aras de estudiar la igualdad efectiva.

La Comisión de Género, en su asesoría, da especial peso a la promoción y formación. En cuanto a la promoción, se han hecho campañas de visibilización y promoción con facilitadores de temas, jueces y periodistas. Igualmente, tanto en las escuelas de bertso como en la formación reglada, se están renovando los materiales didácticos y en los campamentos se trabaja el género. La formación ha generado tres líneas diferenciadas: la primera, dirigida al empoderamiento de la mujer; la segunda, para sensibilizar a los hombres, y la tercera, mixta, para que cooperen hombres y mujeres. Cada línea lleva una velocidad o activación diferente. Las mujeres no necesitan ser convencidas, son conscientes del trabajo que hay. Las otras líneas son más lentas, pero irán descubriendo su propia metodología, si bien es cierto que en la tercera existen más resistencias hacia la perspectiva de género. La línea de las mujeres lleva funcionando siete años y la de los hombres acaba de cumplir dos (Garate 2O2I). En este sentido, Garate (2O2I) entrevista a un conocido versolari, Jon Martín, que ha participado en los dos cursos feministas consecutivos de la bertso eskola feminista para hombres. Martín cita la multitud de temas incómodos y la necesidad de transversalizar el género. Pero hacemos nuestra la pregunta que se hace el versolari entrevistado, qué hacer para desempoderarse y a quién emular en este aprendizaje. Las mujeres versolaris han labrado un camino de empoderamiento con distintos carriles y han invitado a los compañeros versolaris a que se miren sus privilegios, que reflexionen y que se desempoderen. Este tema sería otro paso interesante para el estudio dentro de las metodologías que se están aplicando desde la bertso eskola feminista para hombres, así como realizar un estudio comparativo entre las dos escuelas de bertso feministas.

La Comisión de Género siempre ha estado respaldada por mujeres versolaris y sus iniciativas personales. Muchas veces se han confundido ambas vertientes, ya que las dos trabajan el género. Con todo, las iniciativas de las versolaris son anteriores a la Comisión, lo que ha dado agencia (Kabeer I993) a las versolaris en este ámbito cultural, pasando de ser invisibles a ser parte del jurado y aparecer en público. Así, en la última década, las versolaris han sentido la necesidad de unirse. El primer encuentro de las versolaris con improvisadoras de todo el mundo fue en 2003, en Azkizu, para reflexionar sobre cómo introducir la perspectiva de género en la improvisación oral. Esta experiencia contribuyó a que muchas chicas comprendieran lo que significa ser mujer. Diez años después, en 20I3, volvieron estas iniciativas, donde se quiere concienciar sobre el tema. En 20I4, durante el Día del Bertso, se incorporó la perspectiva de género y las versolaris fueron protagonistas. El año 2or6 se celebró en San Sebastián del II al I6 de julio el encuentro internacional sobre improvisación con jornadas académicas y actuaciones. Un día estuvo dedicado al género.

Con todo, lo más remarcable es cómo surge esta comisión con una visión de empoderamiento, de las iniciativas de las mujeres, de las necesidades de alianzas, para sentirse reconocidas, valoradas y para desarrollar la conciencia feminista. Son conscientes de que aún queda mucho, pero las escuelas de bertsos feministas están en marcha, novedosa experimentación para empoderarse, una metodología para tomar agencia en la hegemónica y masculina plaza. Y una experiencia que se puede extrapolar a otras instancias culturales para contribuir en equidad efectiva. 


\section{Referencias bibliográficas}

AGIRRE, Lorea (2OIO): «Zapia eta txapela Emakume bertsolariak tradizioarekin negoziatzen eta eredu berria sortzen. Negoziazioak eta disonantziak: mututasunetik emulaziora, eta emulaziotik ahots propiora». Trabajo Fin de Máster UPV/ EHU. <http://bdb.bertsozale.eus/uploads/edukiak/ liburutegia/2010\%2 oLorea\%2 OAgirre-zapia\%2 oeta\%2 otxapela. pdf $>$ [Última consulta: junio 2O2I]

AIERDI, Xabier (2007): Bertsolaritza tradizio modernoa. Bilbo: UPV/EHU.

AlBERDI, Uxue (2OI4): «Gorputzak, gorpuzkerak eta gorputzaldiak. Bertsolaritzan». En Gema LASARTE y Amaia Alvarez Uria (eds.) Gorputza eta generoa: Teoria didaktika eta esperientziak. Eibar: UEU, p. I45-I56.

ARtetxe, Miren (2020): «Ipar Euskal Herriko bertso-eskoletako gazteen hizkuntza ibilbideak. Gazte identitateak, hizkuntza mudantzak eta ligitimazio prozesuak». Tesis Doctoral UPV/ EHU <https://addi.ehu.es/ handle/IO8IO/45422> [Última consulta: junio 2O2I]

Aubert, Adriana; Carme GARCíA; Sandra RACiOnero (2009): «El aprendizaje dialógico». Cultura y Educación 2I(2): I29-I39.

BARANDIARAN, Alberto (2OII): Beste larogei urtian. Donostia: Euskal Herriko Bertsozale Elkartea.

BARBOUR, Rosaline (20I3): Los Grupos de discusión en la Investigación Cualitativa. Madrid: Morata.

BEgIRISTAIN, Edurne (2OII): «El bertsolarismo, en el camino de la igualdad». Emakunde 8I: 2.

BOGDAN, Robert; Sari K. BIKLEN (I982): Qualitative research for education: an introduction to theory and methods. London: Allyn and Bacon.

COFFEY, Amanda; Paul ATKINSON (2003): Encontrar sentido a los datos cualitativos. Medellin: Contus.

ColÁs, Maria Pilar; Leonor Buendía (I992): La investigación educativa. Sevilla: Alfar.

ERKIAGA, Nere (2008): «Genero ikuspegia bertsolaritzaren mugimendu barruan». Trabajo de máster, Partaidetza eta Komunitate Garapen Masterra, UPV/ EHU <https://bdb.bertsozale.eus/uploads/liburutegia/xdz5_ld_ooo699.pdf> [Última consulta: junio 2O2I]

FLICK, Uwe (20I4): El diseño de la Investigación. Madrid: Morata.

GARATE, Miren (2O2I): «Feminismoaz gogoeta, bertso eskola moldean». Berria, 202I/o8/24 <https://www.berria.eus/paperekoa/2016/O4O/ooI/2O2I-07-24/ feminismoaz-gogoeta-bertso-eskola-moldean.htm> [Última consulta: junio 2O2I]

GARZIA, Joxerra (20I2): Bertsolaritza. Donostia: Basque Institute.

GARzIA, Joxerra; Jon SARASUA; Andoni EgAÑA (200I): Bat bateko bertsolaritza. Gakoak eta azterbideak. Donostia: Bertsozale Elkartea.

GoETZ, Judith; Margaret LACOMPTE (I988): Etnografía y diseño cualitativo en investigación educativa. Madrid: Morata. 
GonZÁlez AbrisketA, Olatz (20I3): «Cuerpos desplazados. Género, deporte, y protagonismo cultural en la plaza vasca». Revista de Antropología Iberoamericana 8(I): 83-IIO.

Gómez-URdA, Félix (202I): «Una canción sin género: Notas sobre performatividad y agencia en los bertsos de Maialen Lujanbio». AusArt, 9 (I): 197-209.

GorostizA, Ana Isabel, et alii (2020): «Mujeres vascas improvisadoras: las bertsolaris del mundo tradicional (siglos XV-XIX)». Arenal. Revista de historia de las mujeres 27(I): I4I-I72. <http://dx.doi.org/IO.30827/arenal.v27iI.5990>

GREIMAS, Aljirdas Julius (I983): La semiótica del texto, ejercicios y prácticas. Madrid: Gredos.

HABERMAS, Jürgen (I994): Teoría de la acción comunicativa. Madrid: Cátedra.

HERNÁNDEZ, Jone Miren (2006): «Emakume bertsolariak: ahozkatu gabeko identitatea». Kobie: antropologia cultural I2 (2006): 6I-70.

- (2OI4): «Emakume bertsolariak: bertsotik bertsora, hanka puntetan». En Gema Lasarte y Amaia Alvarez Uria (eds.) Gorputza eta generoa: Teoria didaktika eta esperientziak. Eibar: UEU, p. 37-48.

- (20I9): «Algunas instrucciones para abrir la caja negra del conocimiento feminista». Disparidades 74 (I) <https://doi.org/Io.3989/dra.20I9.0I.002.03>

IBARGUREN, Leire (2009): «Nolako umorea egin nahi dugu emakumeok arte eszenikoetan?» Trabajo de Fin de Máster UPV/EHU <http://bdb.bertsozale.eus/ uploads/edukiak/liburutegia/2009\%2oLeire\%2oIbarguren-Emakumeak\%20 oholtza\%2oeta\%2oumorea.pdf>[Última consulta: junio 2O2I]

KABEER, Nalia (I999): «Resources, agency, achievements: reflections and measurements of women empowerment». Development and Change 30(3): 435464.

LARRAÑAGA ARRIOLA, Julen (20I3): Jolas sakona: Txapelketaren prozesu errituala eta bertsolariaren arrazoi sortzailea XXI mendeko agoran. Bilbo: EHU/UPV <http:// www.euskara.euskadi.net/appcont/tesisDoctoral/PDFak/Jexux_Larranaga_ TESIA.pdf> [Última consulta: junio 2O2I]

LARRAÑAGA, Carmen (I994): «Bertsolarismo: Habitat de la masculinidad». Bitarte: revista cuatrimestral de humanidades 4: 29-50.

- (I995): «El Bertsolarismo: una tradición oral transitada por el género-sexo». Cuadernos de Sección. Historia y Geografía 23: 405-425.

- (I997): «Del bertsolarismo silenciado». Jentilbaratz 6: 57-73 <https://www. eusko-ikaskuntza.eus/es/publicaciones/del-bertsolarismo-silenciado/art979I/> [Última consulta: julio 2O2I]

- (2000): «Teatralidad y poética alternativas: El bersolarismo y las mujeres». En Iris M. ZAVAla (ed.): Breve historia feminista de la literatura española (en lengua catalana, gallega y vasca). Barcelona: Anthropos vol. 6, p. 399-424.

LASA OTEIZA, Zaira (2OIO): «Bertsolaria ez da bertsolaria jaiotzen, izatera iristen da. Emakumearen ibilbide bertsolaritzan (I980-2009)». Trabajo de Fin de Máster UPV/ EHU.

LEKUONA, Manuel (I974): «El Bertsolarismo, idazlan guztiak. Ahozko literatura». Kardaberaz bilduma 22: 43-73. 
Limon, Maria Rosario; Juan Antonio CRespo (2002): Grupos de debate para mayores. Guía práctica para animadores. Madrild: Narcea.

MCMillan, James H.; Sally SCHUMACHER (2005): Investigación educativa: una introducción conceptual. Madrid: Pearson.

MitxelenA, Koldo (I988): Historia de la literatura vasca. Donostia: Erein <http:// www.criticalimprov.com/article/view/939/I6Io> [Última consulta: junio 2O2I]

Murguialday, Clara (20I3): Reflexiones feministas sobre el empoderamiento de las mujeres. Barcelona: Cooperació <https://cooperaccio.org/wp-content/uploads/2OI4/o3/Empoderamiento_Cast_web.pdf> [Última consulta: julio 2O2I]

RAPLEY, Tim (2OI4): Los análisis de la conversación, del discurso y de documentos en Investigación Cualitativa. Madrid: Morata.

ReKalde, Itziar; María Teresa Vizcarra; Ana María MaCAZAGA (2OII): «La aventura de investigar. Una experiencia de investigación-acción participativa». Aula Abierta, 39(I): 93-IO3.

- (20I4): «La observación como estrategia de investigación para construir contextos de aprendizaje y fomentar procesos participativos». Educación $x x_{I}$ I7(I):I99-22O.

RodrígueZ, Gregorio; Javier GiL; Eduardo GARCía (I996): Análisis de los datos cualitativos asistidos por ordenador: Aguad y Nudist. Barcelona: PPU.

Rowlands, Jo (I997): Questioning Empoverment. Oxford: Oxfman Publications.

SHOWALTER, Elaine (I983): A Literature of Their Own, British Women Novelists from Brontë to Lessing. Londres: Virago.

SPIVAK, Gayatri Chakravorty (I993): «Can the subaltern speak?» En Patrick Williams y Laura CHRISMAN (eds.): Colonial Discourse and Post-colonial Theory: A Reader. Londres: Harvester Wheatsheaf, p. 66-III.

WHite, Linda (200I): «Orality and Basque Nationalism: Dancing with the Devil or Waltzing into the Future?». Oral Tradition I6(I): 3-28.

WitTIG, Monique (I992): The straight mind and other essays. Boston: Beacon Press. 


\section{Anexo 1. Maialen Lujanbio, canción sin género.}

Tema: Todo iba bien hasta que se ha encendido la luz. Tema que cantó en la final de 2017 .

- Un trago tras otro trago,

- En el interior de nuestras calles

- La fogosidad es lo que el alcohol

- Yel deseo sexual conlleva

- A tu casa, a mi casalo primero, lo de siempre

- Hemos entrado a tu habitación

- Y he aqui lo de después:

- La luz estaba apagada y

- Alli me desnudé del todo

- Y súbitamente se ha encendido

- La más humilde de las luces pequeñas

- Estás mirando a mi cintura

- No soy lo que creias. (bis)

Hasta taparme hemos tenidomuchas risas, muchas bromas

- De boca a boca nos pasábamos

- Los hielos del Gin tonic

- Tenia dos pechos erectos

- Y tú me diste forma

- A pesar de vestir pantalones estrechos

- Sin tacones, ni falda

- Soy esto y aquello estoy tomando

- Algunas hormonas

- Soy hombre y mujer

- Ni mujer, ni hombre

- Mi deseo de no pertenecer a ningún lugar

- Puede que sea la causa de tu desazón. (bis) $* *$.
- Trago bat eta beste trago ba

- Gure kaletan barrena

- Ta piztea da alkoholak eta Sexu grinak dakarrena

- Zure etxera nere etxera

- Betiko gauzak aurrena

- Zure logelan sartu gara ta

- Hara hemen ondorena

- Argia zegon itzalita ta

- Bertan biluztu naiz dena

- Ta bat-batean piztu egin da

- Argi txiki xumeena

- Nere gerrira begira zaude

- Ez naiz uste zenuena. (bis) ${ }^{* * *}$

- Estali arte izan dugunak

- Nahiko irri nahiko broma

- Ahorik aho pasa dugunak

- Gin tonic barruko horma

- Bular parea zut neukan eta

- Zuk eman didazu forma

- Galtza hestuak nebilzkin arren

- Ez takoi ezta ez gona

- Hau ta hura naizHartzen ari naiz

- Zenbait botika hormona

- Gizona eta Andrea nauzu

- Ez Andrea ez gizona

- Nere inon ez egon nahia ote

- Da zure ezin egona. (bis)**.* 